\title{
Il vento nel vigneto di Sgorlon e Il ladro e i cani di Mahfuz: due mondi a confronto
}

\section{Dr. Fawzi Mohamad Abdel Hamid Issa*}

\author{
fawziissa@alsun.asu.edu.eg
}

\begin{abstract}
:
Questo studio mira a mettere a confronto il mondo di Carlo Sgorlon nel suo romanzo Il vento nel vigneto e il mondo di Nagib Mahfuz ne Il ladro e $i$ cani, due mondi in pieno contrasto. I due romanzi, che sono stati scritti e pubblicati quasi nello stesso periodo, parlano dello stesso tema, cioè dell'impresa di due ex carcerati che affrontano la società dopo anni di prigione. Tuttavia ognuna delle due opere riflette il mondo, il pensiero e l'arte del proprio scrittore. Il romanzo di Sgorlon scritto nel 1960, anche se pubblicato in italiano solo nel 1972, racconta l'impresa del protagonista Eliseo che, uscito dal carcere, cerca con determinazione di reinserirsi nella società e di rimettere le proprie radici nelle terre natie, affrontando tutte le difficoltà e le avversità della società e della vita. Grazie alla sua maturazione dentro la prigione e alla sua saggezza, Eliseo riesce alla fine a farsi strada e a conciliarsi con la società e cn se stesso. Al contrario del protagonista sgorloniano, Said Mahran, protagonista de Il ladro e $i$ cani, esce dalla prigione con un unico obiettivo, la vendetta e la rivalsa. Il protagonista di Mahfuz fallisce in tutto, riesce solo a smascherare l'ipocrisia e il tradimento di una gran parte della società. Sono due personaggi insomma completamente opposti che rispecchiano due mondi in contrasto, ma che esprimono perfettamente $\mathrm{i}$ rispettivi pensieri dei loro scrittori.
\end{abstract}

Parole chiave: Sgorlon - Mahfuz - vigneto - ladro - cani

* Docente di letteratura italiana contemporanea-Dipartiemento d'Italianistica Facoltà Al Alsun - Università di Ain Shams

(Il vento nel vigneto di Sgorlon e Il ladro...) Dr. Fawzi Issa 


\section{INTRODUZIONE}

Nel momento in cui Carlo Sgorlon stava concludendo la stesura del suo romanzo Il vento nel vigneto nel 1960, Naguib Mahfuz stava per lanciare il suo libro Il ladro e $i$ cani pubblicato nel 1961. Le due opere sono quindi contemporanee e hanno alcuni punti in comune. Il primo punto di somiglianza consiste nella loro classificazione di due brevi romanzi. L'opera di Sgorlon è di sole 175 pagine con poche diramazioni e pochi personaggi secondari ben delineati dall'autore per servire la trama. Anche quello di Nagib Mahfuz è un romanzo breve, di appena 139 pagine in cui pochi personaggi si stagliano, ma tutti ben delineati, eppure incapaci di salvare il protagonista né da sé stesso né dagli altri.

Le due opere sono accomunate anche tematicamente, poiché raccontano le storie di due ex carcerati appena usciti per la libertà, ma ognuno di loro ha il proprio progetto, molto diverso dall'altro, per affrontare il mondo dopo il carcere. Il primo racconta la vicenda di Eliseo Bastianutti, un ergastolano graziato dopo ventisette anni di prigione, il quale fa ritorno al proprio paese natio nel Friuli, Cassacco, dove era nato anche lo scrittore stesso. Anche Il ladro e $i$ cani racconta la storia di Said Mahran che, dopo quattro anni di carcere, si avvia verso il suo quartiere del Cairo, dove nacque lo stesso Mahfuz.

Attraverso l'avventura del proprio protagonista ognuno dei due scrittori presenta insomma il proprio mondo personale. Infatti, Mahfuz nella maggior parte delle sue opere racconta il Cairo antico con le sue viuzze, i suoi vicoli e la sua gente, soprattutto quel ceto miserabile che ha sempre

\footnotetext{
(Il vento nel vigneto di Sgorlon e Il ladro...) Dr. Fawzi Issa
} 
subito le ingiustizie inflittegli dalla classe governante sia straniera che egiziana. Il ladro e $i$ cani non fa eccezione. Da parte sua, invece, Sgorlon racconta soprattutto il suo mondo regionale, il Friuli, con la sua natura, la sua gente povera e contadina e i suoi paesaggi collinari, perciò, come dice Roberto Damiani, le sue opere:

«Non può che immaginarle nei paesi dove le case sono edificate con i sassi di fiume, nei cortili dall'odore penetrante, nelle distese di terra rubata alla pietra; ad altri personaggi non può affidarle che a contadini, con il loro buonsenso, la loro saggezza, la loro indifferenza nei confronti del potere, il loro senso etico del lavoro». ${ }^{1}$

In questo studio cercheremo perciò di mettere a confronto i due mondi proposti dalle due opere che, come tutte le opere letterarie che fanno da specchio delle società, riflettono due realtà geografiche, sociali e culturali molto diverse.

\section{REALTÀ GEOGRAFICA}

Dal punto di vista geografico, il lettore si trova subito, fin dall' inizio, in due mondi assai diversi l'uno dall'altro: se primo è ambientato in campagna dove la vita procede con un ritmo pacato e sereno, il secondo è ambientato in una delle città più caotiche e più chiassose del mondo.

Alla narrazione de $\mathrm{Il}$ vento nel vigneto fa da retroscena, invece, la campagna friulana dove il paesaggio è un vero protagonista con le sue varietà vegetali e la successione delle stagioni con le quali Eliseo si sente in sintonia. Non mancano così le scene tipiche delle attività contadine durante le varie stagioni, come i campi di 
granoturco e la fila dei carri che trasportano la paglia e il grano:

«La campagna era una vera festa a vederla, il granoturco era già altissimo $\mathrm{e}$ le viti verdi e celesti di solfato. Avevano tagliato il grano, e passando vicino alle trebbie Eliseo vedeva la fila dei carri carichi che aspettavano il loro turno e quelli che andavano via con le balle di paglia e i sacchi gonfi di grano». ${ }^{2}$

Per lo scrittore italiano, la campagna non significa solo la natura, ma è il simbolo di tutta una civiltà, quella contadina, con la sua quietudine, la sua armonia ed i suoi valori. Perciò, lungo tutta l'opera, il lettore viene preso da questo mondo favoloso dove la vita è un susseguirsi di atti armoniosi e dove si vive in sintonia con la natura che risulta molto varia e generosa.

Nella maggior parte delle opere sgorloniane c'è spesso una relazione molto particolare, una specie di simbiosi armoniosa, tra l'Uomo e la natura. Nel nostro caso possiamo notare subito tale sintonia tra Eliseo e la campagna circostante. Basta guardare al modo con cui si mette a potare la vigna di Rita e a sistemare i pali di sostegno, parendo molto esperto e pratico in questi lavori:

«I rami delle viti erano molto lunghi e aggrovigliati. Eliseo li tagliava tutti, allinfuori di tre o quattro dell'ultimo anno, i più lunghi e robusti. Poi li piegava verso il basso, li legava con un vincastro ai fili di ferro. Alla fine, troncava anche le parti superflue del vincastro attorcigliato, perché gli piaceva fare le cose con ordine, e in modo che fossero anche piacevoli da vedere». ${ }^{3}$

Un altro aspetto che si può cogliere anche leggendo il romanzo è il ritmo della vita dei contadini e le attività agricole come la preparazione dei campi alla coltivazione, la

(Il vento nel vigneto di Sgorlon e Il ladro...) Dr. Fawzi Issa 
mietitura e la trebbiatura. Si tratta di attività secolari tramandate da generazione in generazione e esperienze ereditate dagli antenati:

«Eliseo vedeva la fila dei carri carichi che andavano via con le balle di paglia e i sacchi gonfi di grano. Si ricordava delle lunghe soste che faceva da giovane, disteso sul carro a braccia e gambe allargate, e il cappello sugli occhi. Il rumore della trebbia e il polverio della pula erano gli stessi di allora, e anche i contadini che stavano sopra i carri, col fazzoletto sulla bocca e i grandi cappelli sulla testa, che buttavano con la forca $\mathrm{i}$ covoni nel buco della trebbia, compivano ancora gli stessi gesti. Eliseo stava un momento a guardare, e aveva l'impressione che niente da allora fosse cambiato». ${ }^{4}$

Questa armoniosa convivenza con la natura rispecchia la visione molto positiva dello scrittore italiano e la sua concezione particolare della civiltà contadina con tutti i suoi pregi ed i suoi valori. Si tratta di valori che questa civiltà del passato esaltava e che oramai mancano nella nostra civiltà di oggi come il lavoro, la famiglia, la solidarietà sociale..., ecc.:

«La medesima civiltà contadina friulana, che pareva legata da secoli alle sue strutture immutabili, è stata dannosamente contagiata sia dalle ideologie moderne sia dal consumismo, e questo e quello hanno contribuito unicamente al suo deterioramento e alla sua decadenza». 5

Questo spiega anche perché i personaggi sgorloniani sono sempre attaccati al lavoro che considerano un valore in sé stesso, un valore supremo. Nelle opere di questo scrittore troviamo che il lavoro è una fonte di vanto e un motivo di orgoglio. Tale sentimento viene riflesso in questo romanzo dal protagonista che si sente orgoglioso della propria

(Il vento nel vigneto di Sgorlon e Il ladro...) Dr. Fawzi Issa 
partecipazione alla costruzione dell'autostrada che passa per diversi paesi della provincia:

«[...] Pensava che quella strada nasceva anche col suo lavoro, e che dopo finita vi sarebbero passati sopra migliaia o milioni di macchine, di biciclette, di camion. Nessuno avrebbe saputo che vi aveva lavorato anche lui ma a lui bastava averlo fatto, e pensava che in tal modo qualcosa di lui sarebbe sopravvissuto anche dopo la sua morte, in un grande lavoro. Non gli importava se a lavorare si faceva fatica, a lui bastava costruire. Non capiva i giovani che avevano sempre da protestare, e non vedevano l'ora che il lavoro finisse per fuggire dal cantiere, come fosse un luogo maledetto». ${ }^{6}$

D'altronde, lo scrittore friulano ha una visione piuttosto negativa della civiltà moderna, poiché egli crede che l'uomo moderno abbia dimenticato i propri valori e le proprie radici. Perciò, malgrado sia convinto della realtà di un progresso tecnologico e scientifico, egli ha la certezza che tale progresso è ben lontano dal procurare la felicità all'uomo. Gli uomini di oggi diventano sempre più schiavi dei valori materiali e delle nuove scoperte che rovinano la natura e danneggiano la civiltà, il che ha reso la vita più complicata e meno felice, più frustrante e meno tranquilla: «Anche se non dichiarata apertamente, c'è in Sgorlon la consapevolezza degli enormi pericoli che sovrastano l'umanità sovraindustrializzata, superarmata, supertecnologica, lanciata nella corsa pericolosa del consumismo. C'è un pessimismo di fondo in lui nei confronti della storia. Essa è stata sempre in Friuli come altrove, dominata dalle forze irrazionali che sono nell'uomo e nel destino». ${ }^{7}$

Questa visione negativa della civiltà moderna è dovuta anche al fatto che è procuratrice di nuove mode, nuovi

(Il vento nel vigneto di Sgorlon e Il ladro...) Dr. Fawzi Issa 
comportamenti immorali e persino rivoluzioni culturali che sono tutte rivolte verso le illusioni dell'avvenire. Egli vede perciò che questa civiltà e queste rivoluzioni distruggano il patrimonio del passato, molto prezioso e di valore. Tuttavia, egli vede che la civiltà del passato aveva dei lati negativi a cui si deve rimediare, o che vanno eliminati, soprattutto ciò che vi era di ingiusto e di precario:

«Le ragioni sono evidentissime. Come superare quel complesso di forze distruttive che minaccino la nostra civiltà. La stipano di nefandezze e di diletti, e ci fanno intravvedere all'orizzonte persino la fine della vicenda umana sulla terra, se non ripristinando il sentimento del sacro? Se non rimettendo pazientemente vicino i frammenti di qualcosa che è andato spezzato e guastato negli ultimi secoli?». ${ }^{8}$

Nel romanzo dello scrittore egiziano, invece, la vicenda ha luogo al Cairo degli anni Cinquanta ed i primi anni Sessanta, con i suoi mille volti che portano i segni del passato, ma anche lo sguardo verso il futuro. Il Cairo con le sue contraddizioni architettoniche e sociali dove, da un lato, troviamo i quartieri signorili con ville e case lussuose, e dall'altro i quartieri popolari dai vicoli lunghi e stretti e dalle strade dove circolano $i$ tram e dove molte case hanno i muri scrostati e fitti di crepe:

«Le taverne sono chiuse e non rimangono che $\mathrm{i}$ vicoli per ordire complotti. Di tanto in tanto il piede cavalca una buca che segna il marciapiede come un trabocchetto. Lo sferragliare delle ruote dei tram rimbomba come uno scroscio di ingiurie; le diverse grida dei mercanti si armonizzano come le esaltazioni delle immondizie. Ah, giuro che ti odio!». ${ }^{9}$ 
Nagib Mahfuz ha scelto così con cura la topografia del suo racconto, perciò troviamo che Said Mahran si muove come in un triangolo i cui tre angoli sono la casa di Shaykh Gunaydi, quella della prostituta Nur e il caffè del padron Tarzan:

«L'ambientazione è quella di una città, il Cairo, divisa in due tra nuove ricchezze e perenne squallore, alle ultime propaggini del deserto: un ultimo bar prima della sabbia ed un'ultima casa prima del cimitero.

In questo mondo dominato dalle tenebre, solo due flebili luci: una donna di nome Nur ed un vecchio maestro spirituale. Ma anche l'amore e la religione non possono in alcun modo lenire l'angosciata solitudine dell'individuo, costretto a vivere in un mondo dominato dall'ingiustizia e dal tradimento». 10

Si tratta di un triangolo che simboleggia le strade rintracciate dallo scrittore per il suo protagonista che deve scegliere lui quella da percorrere. Tuttavia, né l'amore (la casa di Nur) né la fede (la casa dello Shaykh) sono in grado di aiutare Mahran a realizzare la rivalsa molto ambita. Non gli manca dunque che la strada del crimine (il caffè del padron Tarzan), il percorso scelto dai ripudiati fin dall' antichità. ${ }^{11}$

\section{REALTÀ SOCIALE}

Le due opere hanno contesti sociali molto differenti. Nel suo romanzo Sgorlon descrive la società novecentesca soprattutto nei piccoli centri e nei villaggi del Friuli. Si tratta di una società che porta le sembianze della civiltà contadina oramai in via di sparizione. In vari punti dell'opera lo scrittore, infatti, ci descrive una società che conserva ancora 
certi valori come la solidarietà e l'unità familiare. Questo risulta evidente quando Eliseo, in cerca di una abitazione, va all' indirizzo indicatogli dalla ragazza dell'osteria, a chiedere se la stanza fosse disponibile. Arrivato là, trova questa scena, caratteristica della vita campagnola, con tutta la famiglia riunita intorno alla tavola, e al focolare domestico, con il padre in mezzo:

«Disse "compermesso" ed entrò nella cucina, dove attorno alla tavola erano seduti due giovanotti, un uomo e una donna piuttosto anziani, e alcune ragazze da marito. Sulla tavola c'era un piatto rotondo con fette di salame, un grosso pane, un bottiglione di vino nero parecchi bicchieri. Nella stanza faceva un bel caldo, un fascio di viticci bruciava crepitando sul focolare. Tutti, vedendo l'intruso, si voltarono dalla sua parte. Guardandolo con uno stupore un po' seccato. I discorsi si fermarono, e tutti aspettarono, restando seduti, che l'uomo dicesse ciò che voleva». ${ }^{12}$

Un'altra caratteristica di questa società è quella di rispettare la natura e consacrare il lavoro. Perciò nelle comunità contadine, si nota un forte legame con la terra e un apprezzamento del lavoro manuale. Qui infatti troviamo la gente che lavora i campi seguendo il ritmo delle stagioni, a partire dall'aratura e la semina dei campi fino alla raccolta $o$ la mietitura, malgrado la fatica e lo sforzo richiesti da questo lavoro:

«Iolanda gli aveva scritto dove si trovava la sua casa, ma lui adesso non riusciva a orientarsi con tutte le nuove costruzioni. Dai cortili veniva odore di stalla; vide un paio di contadini che caricavano il letame sulla carriola di legno a grandi colpi di forca, e lo trasportavano nel mucchio dietro la casa». ${ }^{13}$ 
Ma questa società era anche contrassegnata da alcuni aspetti negativi come la miseria e le ingiustizie sociali: pochi erano infatti quelli che godevano di grandi proprietà mentre la maggior parte della popolazione soffriva delle malattie e viveva in assoluta povertà. Questo fatto si rende chiaro in numerose parti del romanzo come quando Eliseo, povero com'è, si dimostra soddisfatto della somma presa in cambio al lavoro all'autostrada, mentre gli altri operai si lamentano del carovita e si dichiarano completamente insoddisfatti delle loro paghe:

«"Allora è proprio vero quello che dicono di te"

Cioè?".

"Che non sei del tutto a posto. Come fai a dire che siamo ben pagati? Fortuna che stiamo tutti in campagna, che bella o brutta abbiamo tutti una casa, un pezzo di orto e un po' di pollame, se no non sapremmo come tirare avanti..."

Eliseo non rispose, e anzi si pentì di aver parlato. Capì che i soldi che gli davano bastavano a lui che era solo e viveva di niente, ma che se avesse avuto famiglia sarebbe stato fresco...». ${ }^{14}$

Questa miseria e questa povertà stanno alla base anche dell'ignoranza in cui vive il popolo, soprattutto nei ceti più bassi come quello degli operai che lavorano nella costruzione dell'autostrada. Questo si vede dal discorso fatto tra il protagonista e uno degli operai la cui moglie sta male ed è stata operata più volte e il marito non sa nemmeno di che si tratta:

«Uno di loro era tutto preoccupato perché la moglie doveva entrare in ospedale per farsi operare.

"Di cosa si tratta?" domandò Eliseo. 
"Me lo hanno detto, ma non mi ricordo. E un male piuttosto raro. Mah!".

"Vedrai che andrà tutto bene. Oggi fanno miracoli".

"Speriamo. Però, vedi, è la terza volta che deve farsi tagliare in quattro anni. È molto giù, e neanche il cuore lo ha molto robusto. Mah!"».15

Ma se in campagna viveva gente così misera e così povera, il romanzo ci rispecchia anche altre classi sociali oltre ai contadini e agli artigiani. Questi ceti variano dai piccoli proprietari alla ricca classe delle città che ci tiene a fare $\mathrm{i}$ collezionisti di antichità e vive in residenze lussuose, il che ha favorito l'avvento di nuovi mestieri e nuove attività come quello dello straccivendolo o quello dell' antiquario che vanno in cerca di oggetti antichi che rivendono in città:

«ll Nino gli mostrò quali cose si potevano rivendere con maggior convenienza. C'erano a Udine negozi e rivenditori che compravano mobili antichi, oggetti di rame, di ottone o di ferro battuto, e allora non si parlava più di guadagni da straccivendolo, ma da antiquario. Gli disse che adesso molta gente aveva la mania di quelle cose, e più ruggine e più tarli si portava in casa, più era contenta. Era il momento d'oro per quel tipo di commercio, e bisognava sfruttarlo prima che gli antiquari di città cominciassero a battere le campagne da soli.» ${ }^{16}$

La società descritta nell'opera di Mahfuz è, invece, la società egiziana in generale e quella cairota in particolare dopo la rivoluzione del 1952, una società caratterizzata dalle contraddizioni più acute. Da una parte troviamo gente benestante che abita ville o palazzi e case signorili sul Nilo come l'abitazione di Rauf Olwan, dall'altra ci sono persone povere che abitano nei quartieri limitrofi della città come la

(Il vento nel vigneto di Sgorlon e Il ladro...) Dr. Fawzi Issa 
casa di Nur che dà sul cimitero, o quella dello shaykh Gunaydi a Darrassa al limite del deserto:

«Mentre si avvicina percorrendo la strada delle colline, egli guarda la porta aperta. Per quanto ricordi, è sempre stata spalancata. È un luogo di buoni ricordi situato nel quartiere di Darrassa, tra le due bracci del Muqattam. Il terreno sabbioso pullula di bambini e di insetti. Egli ansima per la fatica e l'emozione. I suoi occhi cercano avidamente le bambine della collina, che ripara dai raggi del sole ormai obliqui"»». ${ }^{17}$

Tale contraddizione risulta molto evidente paragonando tra il quartiere popolare dove viveva il protagonista prima di entrare in prigione e dove attualmente vivono Alish e Nabaweyya con la figlia Sanà da una parte, e i quartieri signorili del Cairo dove Said Mahran sceglieva accuratamente le sue vittime prima di essere catturato e dove vive ora Raùf Olwan dall'altra. Nel primo vive la gente più misera della popolazione, un miscuglio delle persone più svariate per appartenenza, per professione e per ideologia, ma tutte accumunate dalla miseria e dalla poca cultura:

«... Entrano nella sala e si accomodano sparpagliatamente sui canapè e sulle sedie. Vengono aperte le finestre, e nella stanza irrompono luci e mosche insieme. Il tappeto blu cielo è disseminato si segni neri, le bruciature dei mozziconi di sigaretta. Con le mani appoggiate sopra un bastone nodoso, Alish li squadra come fa un ritratto appeso al muro. Quanto all'agente, è seduto a fianco di Said e inizia a giocherellare con i grani del rosario. Entra Alish Sedra: indossa un'ampia gallabya rigonfia come una botte intorno al suo corpo sormontato da un volto tondo con il doppio mento, prominente al punto da formare quattro pieghe, e un grosso naso con le narici schiacciate». ${ }^{18}$

(Il vento nel vigneto di Sgorlon e Il ladro...) Dr. Fawzi Issa 


\section{SFONDI POLITICI}

Sul livello politico le due opere hanno due sfondi assai diversi. Ne Il vento nel vigneto notiamo il disinteresse del protagonista nei confronti della politica, cosa che rispecchia soprattutto la concezione sgorloniana abbastanza negativa della politica. Tuttavia, allo sfondo della vicenda ci si accorge del fermento politico della popolazione, soprattutto dei giovani entusiasti che lanciano degli slogan e ripetono termini del linguaggio politico che Eliseo non conosce perché apparsi dopo la liberazione quando lui era in prigione:

«Lo accusavano di qualunquismo e di non avere coscienza di classe. Eliseo accettava le accuse in silenzio e con apparente indifferenza, ma in realtà si vergognava del suo assenteismo. Non capiva la passione degli altri e si rendeva conto che accadeva, almeno in parte, perché lui non c'era quando succedevano i fatti che l'avevano acceso». ${ }^{19}$

Nel romanzo di Mahfuz, invece, lo sfondo politico gravita intorno al socialismo, molto esaltato negli anni Cinquanta e Sessanta, dopo la rivoluzione del 1952, durante l'epoca nasseriana, anche se il pensiero socialista è stato, come dice Shafiq Farid, una componente ideologica dello scrittore fin dal 1938. La sua tendenza verso il socialismo sarebbe dovuta a tre fattori principali quali la sua coscienza delle sofferenze della società egiziana dell'ingiustizia nella distribuzione della ricchezza, la sua condizione di studente, per lungo tempo, di Salama Musa e la sua consapevolezza che il socialismo è la fine inevitabile di ogni progresso intellettuale, morale e sociale nel mondo di oggi. ${ }^{20}$

Dato ciò l'autore ci presenta il suo protagonista come un ladro convinto di un'idea che difende anche combattendo,

(Il vento nel vigneto di Sgorlon e Il ladro...) Dr. Fawzi Issa 
un ladro che ruba essendo convinto della moralità del proprio comportamento, per recuperare con la violenza la giustizia violata:

«Giunto al punto Abbas, egli siede su una banchina di pietra e si accorge per la prima volta di ciò che lo circonda. Poi come rivolgendosi all'oscurità, con voce forte e chiara dice: "La carità non si fa pregare... Non perdere tempo e approfitta dell'effetto sorpresa!". Non hai motivo di esitare, si tratta del tuo mestiere: è nobile e soprattutto onesta, quando ha come bersaglio il suo padre spirituale». ${ }^{21}$

Non si tratta insomma di un ladro di quelli abituali nella nostra vita quotidiana. È una vittima degli imbrogli di Raouf Olwan che l'ha convinto che il furto è una specie di lotta contro l'ingiustizia sociale. Quante volte Olwan ha promosso questa idea come concetto politico quando era ancora uno studente povero. Ed ora dopo essere diventato ricco, padrone di una villa, diventa un difensore della proprietà privata e un nemico palese di Said in quanto minaccia a questa proprietà. ${ }^{22}$

\section{AFFINITÀ E DIVERGENZE TRA I DUE PROTAGONISTI}

Nonostante siano diversi da vari punti di vista, il primo punto in comune tra $\mathrm{i}$ due protagonisti consiste nell' inizio difficoltoso delle loro imprese: sin dal primo momento di libertà i due uomini vengono contrastati persino dal clima poco mite e poco favorevole, come se la natura stessa non guardasse di buon occhio il loro ritorno alla libertà. Eliseo al suo ritorno, appena sceso dal treno, trova un freddo molto forte e insopportabile.

(Il vento nel vigneto di Sgorlon e Il ladro...) Dr. Fawzi Issa 
«"Stazione di Tricesimo" disse due volte il controllore. Poi aprì lo sportello e saltò sul marciapiede.

L'uomo gli andò dietro, facendo a uno a uno gli scalini del predellino. Ma non c'era anima viva. Faceva molto freddo. Il vento girava attorno alla stazione e fischiava tra i rami dei gelsi. Presto il treno ripartì, con impeto stridente, e l'uomo stette a guardarlo, finché le sue luci sparirono dietro una curva lontana. Scosse la testa e si avviò verso lo stradone, cercando di camminare più svelto che poteva per scaldarsi». ${ }^{23}$

Ma se Eliseo soffriva dal freddo e dal vento gelido Said Mahran soffriva, invece, dall' aria soffocante e dal caldo afoso caratteristici del Cairo nei giorni d'estate:

«Di nuovo egli respira aria di libertà; ma l'atmosfera è soffocante, come impregnata di polvere, e il caldo insopportabile. Ritrova l'abito blu e le scarpe da ginnastica, ma non v'è nessun altro ad aspettarlo. Ecco riapparire il mondo, ecco dischiudersi il grande portone del carcere su segreti senza speranza.

Le strade sono come oppresse dal sole, le automobili sembrano impazzite, i passanti e le persone sedute ai tavoli, le case e i negozi... e nessuno che accenni un sorriso.

Egli è solo, avvilito. In carcere, ha perduto quattro preziosi anni della sua vita e per un altro anno ancora verrà segnato a dito». ${ }^{24}$

Eliseo Bastianutti e Said Mahran sono accomunati anche dalla difficoltà a reintegrarsi nella società e dallo sguardo negativo dei compaesani nei loro confronti. La loro accoglienza da parte della gente era molto fredda, e poco ospitale. Per la strada verso il suo paese, Eliseo viene riconosciuto dalle donne che risciacquano i secchi del latte nelle fontane, e quando si avvicina tacciono le voci e si

(Il vento nel vigneto di Sgorlon e Il ladro...) Dr. Fawzi Issa 
riprendono alle sue spalle appena allontanato. Anche quando arriva alla prima osteria e dopo aver trovato alloggio e cominciato a sentirsi il piacevole caldo della stufa, viene riconosciuto da un avventore del locale e si trova costretto a prendersi la valigia e andare via:

«L'uomo capì subito che parlavano di lui. Provò un acuto disagio e decise immediatamente di andarsene, per quanto fosse stanco e li, vicino alla stufa, si sentisse molto meglio. Pagò il vino e uscì. Gli sembrò che adesso facesse meno freddo di prima, certo perché era stato al caldo e tra le case il vento si sentiva di meno». ${ }^{25}$

Persino il padrone della seconda osteria, malgrado la sua convinzione che Eliseo è un buon uomo, non ha voluto dargli la stanza vuota nel retro della bottega che gli aveva promesso. Egli era certo che l'uomo era onesto ed era un buon lavoratore soprattutto dopo la prova che Eliseo ha dato costruendo alla perfezione il muretto voluto dall'oste. Tuttavia, quando gli ha chiesto la stanza, quest'ultimo si è scusato cortesemente dicendo che l'osteria avrebbe subito danni perché la gente non smette mai di parlare:

«"E voi non fidate di me...".

"Non è questo" disse l'oste "neanche per sogno. Per quello che io so, voi siete un galantuomo e un lavoratore. Non è per me, ma per la gente. Non sapete com'è fatta la gente di paese, cosa può nascere quando si mettano a parlare di una cosa. L'osteria può perderci molto. Dovete capirmi" $\gg .{ }^{26}$

A sua volta Said Mahran viene accolto con ipocrisia da parte degli abitanti del quartiere, tranne pochissimi suoi amici, complici o vecchi collaboratori:

«Si ferma ad aspettarlo, poi si stringono la mano e si abbracciano, mentre un tenue sorriso dissimula $\mathrm{i}$ veri 
sentimenti. Il farabutto ha sempre i suoi complici. Tra poco, lui vedrà che cosa cela quest'accoglienza. Forse te ne stai nascosto, guardando attraverso le fessure delle persiane come fanne le donne, o Alish». ${ }^{27}$

Ma la sua grande delusione è stata ricevuta dal suo amico Raouf Olwan, il giornalista che era la sua guida e il suo maestro spirituale in passato. Il padre di Said Mahran faceva il portiere nella palazzina degli studenti dove il protagonista conobbe Raouf quando era ancora studente all'Università. Olwan convinse il padre di Said di iscriverlo a scuola e impresse nel fanciullo l'amore per i libri e la lettura. Non solo, ma Olwan difese Said quando quest'ultimo commise un furto, e lo incoraggiò a rubare i ricchi perché "il ladro non fa altro che recuperare quanto i ricchi hanno rubato agli altri'.

Quando Mahran si rivolge a Raouf per chiedergli di aiutarlo a trovare un lavoro e una casa egli viene deluso dalle parole del suo vecchio maestro spirituale che gli paiono ormai prive di significato. Gli amici di un tempo lo deludono e lo scansano, incontra enormi difficoltà, da ex carcerato. In breve, si convince che l'ultimo senso residuo della sua esistenza risiede proprio nella vendetta, e si dedica a prepararla accuratamente:

«Rauf abbassa gli occhi come se queste parole lo avessero persuaso, ma appare evidente che il suo viso non ha più un'espressione rilassata. Riprende a parlare, ma il tono è quello di chi ha premura di troncare un incontro:

"Said, oggi non è più ieri. In passato eri un ladro e un amico per le ragioni che ben conosci. Ma i tempi cambiano e se torni a rubare, per me sarai solamente un ladro!"

(Il vento nel vigneto di Sgorlon e Il ladro...) Dr. Fawzi Issa 
Said si alza di scatto, trovandosi improvvisamente di fronte all'aspetto più crudele della disperazione. Trattiene ogni emozione per mezzo della sua ferrea volontà, si risiede e, con voce calma, dice:

"Trovami un lavoro che possa andare bene per me!"». ${ }^{28}$

\section{TRATTI FISIONOMICI E CARATTERIALI}

Nelle due opere la narrazione è piuttosto snella e la trama è imperniata intorno al protagonista, mentre gli altri personaggi secondari, che sono pochi, fanno da coro o contrappunto per il personaggio principale. Ne Il vento nel vigneto troviamo che il filo conduttore di tutta l'opera dipende unicamente da Eliseo, mentre ne Il ladro e $i$ cani tutta la vicenda è imperniata intorno a Said Mahran. Fin dalle prime pagine dei due romanzi ci troviamo di fronte a due personaggi che si possono considerare opposti dai punti di vista fisionomico e caratteriale.

Sgorlon ritrae il suo protagonista come un uomo robusto, alto e forte. La descrizione di Eliseo non viene, però, fatta direttamente dall'autore, ma il lettore la deduce da alcune situazioni e dettagli forniti pian piano lungo il romanzo. I tratti fisionomici del personaggio vengono forniti poco alla volta durante la narrazione. Per esempio, attraverso il paragone tra Eliseo e il Marito di Rita veniamo a sapere che lui è molto alto di statura:

«[...] Eliseo si avvicinò e osservò anche lui. Nella foto

Rita aveva accanto a sé un uomo che la superava di più di mezza testa.

"Vostro marito?" disse Eliseo.

Rita fece cenno di sì.

"Che pezzo di uomo".

"Era alto uno e novanta".

(Il vento nel vigneto di Sgorlon e Il ladro...) Dr. Fawzi Issa 
"Proprio come me" aggiunse Eliseo, ma subito si pentì di averlo detto, e di nuovo pensò che doveva essere offensivo per la donna paragonarsi in qualche modo al morto». ${ }^{29}$

Il lettore riesce anche ad immaginare la robustezza del protagonista quando Rita insiste a dargli, in cambio al suo lavoro nella vigna, il vestito del suo defunto marito. Qui scopriamo che tale vestito andava allargato un po' per essere perfetto e alla misura di Eliseo:

«Eliseo si arrese, e andò di sopra a provarlo. Gli andava quasi bene, bastava allargarlo un po', rifare qualche cucitura. Era un bel vestito a quadri, e quasi nuovo, anzi a uno che non se ne intendeva poteva parere nuovo del tutto». ${ }^{30}$

Lo scrittore mette in risalto poi la robustezza e la forza del suo protagonista verso la fine dell'opera nella scena in cui succede lo scontro tra Eliseo e il conduttore del rullo. Questi, un giovanotto orgoglioso e troppo superbo, aveva provocato Eliseo perché aveva lasciato la pala sull'orlo della strada e il rullo l'aveva rovinata. Malgrado che Eliseo abbia riconosciuto il proprio errore e la sua disposizione a pagare $\mathrm{i}$ danni il giovanotto continuò e gridare, insultare l'uomo e bestemmiare. Nonostante tutti i tentativi degli altri operai di calmare il giovanotto, questi non voleva saperne e trovava inaudito che "un manovale assunto per pietà osasse rispondere a lui, che è uno specializzato e lavorava nella ditta da anni". Eliseo si tratteneva e non voleva che succedesse come era successo in passato, ma con l'insistenza del giovane lo scontro era inevitabile:

«Eliseo alzò le braccia e il giovanotto gli colpì il destro con il manico di legno. Fece in tempo a vibrare un colpo

(Il vento nel vigneto di Sgorlon e Il ladro...) Dr. Fawzi Issa 
solo, perché Eliseo gli era già addosso. Lo afferrò per la vita, e lo sollevò. Tutti trattennero il fiato per paura che succedesse qualcosa di brutto, e restarono a guardare pieni di paura, soggiogati dalla forza enorme di Eliseo. Il giovanotto gridando scagliò via la pala e cercò di afferrare il collo dell'avversario, ma l'uomo lo buttò a rotolare sull'erba del campo vicino alla strada. Poi prese la pala, l'appoggiò al ginocchio e ne spaccò il manico come fosse uno stecco». ${ }^{31}$

Da parte sua, Mahfuz non si dilunga nella descrizione fisica di Said Mahran di cui fornisce solo poche nozioni, ma indispensabili, perché il lettore possa immaginare la sua corporatura minuta e snella, idonea alla sua illecita attività come ladro abituato ad arrampicarsi sui tubi e rubare le abitazioni:

«Osservandolo attentamente, con i suoi occhi brillanti e il corpo slanciato e vigoroso, Said sembra una tigre che tenda l'agguato a un elefante. Egli riesce solo a ripetere le parole di Alish:

"Solo chi lo merita dev'essere castigato"».. 32

Dal punto di vista caratteriale, invece, i due scrittori hanno descritto perfettamente i loro protagonisti, anche se servendosi di tecniche diverse. Nel romanzo italiano lo scrittore ci presenta Eliseo come un eroe dotato di tutte le caratteristiche indispensabili per la realizzazione del proprio obiettivo come ha forza morale, la tenacia e la pazienza. Il personaggio subisce anche uno sviluppo positivo nel corso della vicenda e la sua personalità cambia completamente dopo la prigione. Prima era una persona orgogliosa, superba e prepotente che non accettava la minima offesa:

«Ne ero sicuro. Lo sanno tutti. Sono stato in galera più di metta della mia vita, che è già lunga. Questo è 
successo solo perché ero violento e superbo. Ho ucciso chi mi aveva offeso, prima. È una storia complicata, Riccardo, non so se potresti capirla" $\gg .{ }^{33}$

Lo scrittore sintetizza il cambiamento totale della personalità di Eliseo, attraverso la tecnica del flash back e i ricordi che, di tanto in tanto, il protagonista ha durante la narrazione come nel brano seguente:

«Da quanto tempo non si trovava in una famiglia? E del resto, anche prima che lo portassero via, a casa sua ci stava meno che poteva. Quando non era via col carro, con il sole o con la luna, era sempre in giro con gli amici o le ragazze. Le mura di casa gli pesavano addosso, allora, e per sentirsi bene aveva bisogno di essere in un locale pubblico e di avere attorno tanta gente. Gli piaceva mettersi in mostra. Tracannava mezzo litro di vino senza staccare le labbra dal boccale, batteva i pugni sul banco, non sopportava la più piccola offesa. Sua madre si lagnava con lui perché era sempre fuori, ma erano parole che lui non sentiva nemmeno. Si chiese con stupore come mai potevano piacergli queste cose, allora, come se non si riconoscesse nei propri ricordi...» ${ }^{34}$

Da parte sua, malgrado la descrizione fisionomica molto snella del suo protagonista, lo scrittore egiziano ha ritratto perfettamente il suo personaggio dal punto di vista caratteriale. Mahfuz fa di Said Mahran uno di quegli eroi negativi che sono, tuttavia, in grado di emanare un fascino contagioso, tanto da capovolgere i valori della società e le intenzioni moralistiche più tradizionali:

«E, chinandosi verso Said, Tarzan sussurra:

"Non fermarti più di una notte nel medesimo luogo."

Al che, il trafficante d'armi dice:

"Scappa nell'Alto Egitto!"

Said Risponde:

(Il vento nel vigneto di Sgorlon e Il ladro...) Dr. Fawzi Issa 
"Non conosco nessuno, da quelle parti."

Di rimando il mercante afferma:

"Molti parlano di te con grande ammirazione"». ${ }^{35}$

Questa figura eroica del protagonista agli occhi della gente viene anche confermata da Nur che, oltre ai cibi più deliziosi e i giornali, gli porta anche le notizie della gente che parla di lui come di un eroe:

«Nur si spoglia: ora s'indossa soltanto una camicia trasparente. Le nari di Said avvertono un odore di trucco e sudore frammisti. Ella dice:

"La gente parla di te come di Antara, ma ignorano il nostro tormento"». ${ }^{36}$

Insomma, Mahfuz ci presenta il suo protagonista come antieroe, capace però di accattivare la simpatia e l'immedesimazione del pubblico. Said Maharan infatti, nonostante sia solitamente portatore di tratti negativi e malgrado che possa assumere il ruolo di personaggio cattivo, non viene considerato tale dal pubblico, poiché ha commesso i suoi crimini solo per altre ragioni, nascondendosi dentro una personalità originariamente positiva.

Lo scrittore, con un certo distacco realistico, lascia al suo protagonista tutta la libertà di scegliere la strada da percorrere nella vita, e gli dà due alternative completamente opposte. Perciò Said Mahran trovava rifugio in due posti diametralmente opposti, ma molto significativi dal punto di vista morale e simbolico: la casa della prostituta Nur e quella dello shaykh sufi Alì Gunaydi, anche se lui alla fine sceglie una strada diversa, quella della violenza e della perdita. Non è dunque strano che alla fine lui scappa nel cimitero che ha qua un valore simbolico molto importante: è il simbolo della morte e la destinazione finale dell'essere umano. Infatti, il

(Il vento nel vigneto di Sgorlon e Il ladro...) Dr. Fawzi Issa 
ladro trova la sua fine fatale dopo essere stato assediato e circondato dalla polizia e dai cani:

«Immediatamente dopo il suo richiamo, ode in lontananza l'abbaiare dei cani che, nel silenzio, ricorda un susseguirsi di scoppi. In preda al panico indietreggia. Subito si nasconde tra le tombe, mentre il guaiolare degli animali si avvicina sempre più. Appoggiandosi su una lapide, punta la pistola in direzione delle tenebre, oramai certo dell'approssimarsi della fine. Ma, a vanificare ogni speranza, ecco giungere i cani. Almeno per il momento, $\mathrm{i}$ farabutti sono salvi. La sua vita gli lancia un'ultima parola, rammentandogli la propria inutilità». ${ }^{37}$

\section{PERSONAGGI SECONDARI}

Ognuno dei due scrittori ha ritratto pochi personaggi che gravitano intorno al protagonista ma che sono molto importanti e indispensabili per la trama e lo sviluppo della vicenda. Ognuno di questi personaggi rappresenta un archetipo che serve ad esprimere la filosofia, il pensiero ed $\mathrm{i}$ concetti del proprio scrittore.

$\mathrm{Ne}$ Il vento nel vigneto oltre a Eliseo ci sono sua sorella Iolanda, Rita e Giovanna di cui parleremo più avanti, oltre all'oste, Nino, il conduttore del rullo e il figlio di Rita, Riccardo.

L'oste viene presentato come una persona saggia e gentile, sempre disponibile ad aiutare il protagonista senza, però, rischiare troppo. Egli infatti dà ad Eliseo la prima opportunità di lavoro, affidandogli il carico di costruire il muro nella cantina. E, malgrado che non volle dargli la stanza vuota nel locale, perché sapeva che la gente del paese non smetteva di parlare cosa che avrebbe avuto un esito 
negativo sul locale, continuò ad aiutare il protagonista, proponendolo a chi cercava un lavoratore:

«... Quando sentiva parlare di un lavoro qualsiasi, l'oste

diceva ogni volta: "Perché non chiamate Eliseo?".

"L'ergastolano?".

"Perché? Non vi fidate?".

"Non è perché non mi fido. Ma così... mi sembra una cosa".

"Sciocchezze. Lavora bene e costa poco"». ${ }^{38}$

L'oste continua a svolgere questo ruolo positivo nei confronti del protagonista, suggerendogli per esempio il falegname dal quale Eliseo lavora a pezzo, quando manca il lavoro nei campi, soprattutto durante l' inverno. Non solo, ma è stato molto d'aiuto nell'acquisto della falegnameria facendo da mediatore e convincendo il proprietario a venderla a Eliseo che diventa padrone e comincia a lavorare in proprio:

«L'oste fu lieto di prestarsi alla mediazione, e domandò al falegname se non era stufo di stare a riempire la gola di segatura tutto il giorno, tanto si sapeva che non era morto di fame e che il pane non gli mancava. Il vecchio rispose che non vedeva l'ora di liberarsene. Non aveva neanche a chi lasciarla in eredità, e così l'avrebbe data via magari per un boccone di pane, perché ormai non riusciva più a sopportarla». ${ }^{39}$

Anche Nino, lo straccivendolo, rappresenta l'archetipo della persona attiva che si è creata dal nulla, grazie alla sua intelligenza e al suo impegno lavorativo. All'inizio Eliseo conosce Nino come cliente comprando da lui alcune cose. Poi Nino diventa una tappa molto importante nella vita del protagonista e nella sua affermazione. L'autore lo ritrae

(Il vento nel vigneto di Sgorlon e Il ladro...) Dr. Fawzi Issa 
fisionomicamente con poche parole, ma dal punto di vista caratteriale gli dedica maggiore spazio, a diverse riprese: «Era un giovanotto ben piantato, con una cicatrice obliqua sulla guancia. Di famiglia poverissima, non gli avevano insegnato nessun mestiere, se non l'arte di contare solo su di sé. Appena finito il servizio militare era tornato a casa con quella cicatrice e una gran voglia di fare soldi, e si era messo a comprare e a rivendere ogni genere di cose. Parlava poco, ma aveva sempre la battuta pronta». 40

Il conduttore del rullo poi è l'esempio del giovane superbo e orgoglioso che ricorda Eliseo nella sua giovinezza, ma ora rappresenta il contrappunto dell'Eliseo attuale, calmo, contemplativo e saggio. Questo personaggio serve a mettere alla prova il protagonista che riesce ad autocontrollarsi malgrado le provocazioni molto forti da parte del giovane, e ciò risulta molto chiaro dallo scontro avvenuto durante il lavoro nell'autostrada.

Riccardo invece è l'archetipo del fanciullo amabile, molto vivace, svelto e intelligente, che malgrado sia d'ostacolo al matrimonio tra Rita e Eliseo, funge anche da anello di congiunzione fra loro. Egli infatti svolge un ruolo di aiutante al protagonista a cui è molto attaccato e a cui vuole molto bene. Da parte sua, Eliseo lo considera come il figlio mai avuto. Dato ciò troviamo che si trovano bene insieme, come se fossero padre e figlio:

«Eliseo lo mandò a farsi prestare un paio di forbici, e Riccardo partì di corsa per tornare dopo dieci minuti, sempre correndo. L'uomo gli insegnò come si tagliavano i tralci superflui e si curavano gli altri. Il ragazzo imparò subito, ma lavorava sempre vicino a lui per chiedergli consigli. Ben presto non fu nemmeno necessario parlare,

(Il vento nel vigneto di Sgorlon e Il ladro...) Dr. Fawzi Issa 
Riccardo gli indicava un ramo, ed Eliseo rispondeva "si" o "no" con un gesto della testa, come tra loro ci fosse un'antica abitudine a stare insieme». ${ }^{41}$

$\mathrm{Ne}$ Il ladro e i cani ci sono anche personaggi secondari di primo piano come Nur e Nabawiyya, di cui parleremo più avanti, oltre a Rauf Olwan, lo shaykh Gunaydi, Alish e il padron Tarzan.

Rauf Olwan è l'esempio della persona opportunista che prima rappresentava lo studente rivoluzionario cosciente dell' ingiustizia sociale e convinto della necessità di costruire un nuovo mondo più giusto. Tuttavia, il protagonista scopre poi che il suo maestro spirituale che gli aveva insegnato di portare la conoscenza in una mano e la pistola nell'altra è già cambiato e che le sue parole sono ormai prive di significato. Said Mahran perciò vede in lui un traditore più grave di Nabawiyya e Alish, perché questi hanno tradito solo lui, mentre Olwan ha tradito le masse, i suoi lettori e i suoi allievi come lui, e si è spostato nell'altra sponda, facendo degli slogan rivoluzionari solo maschere dietro alle quali si nascondeva, perciò tutti i tradimenti sono perdonabili tranne questo. $^{42}$

Lo shaykh Gunaydi è l'archetipo dello shaykh sufi mistico, intento all'adorazione di Dio con i suoi discepoli. Il sufismo era notevolmente diffuso in Egitto fino alla metà del Novecento e aveva grande influenza sui ceti popolari. Perciò la casa dello shaykh rappresenta la fede, la religiosità e la spiritualità in una società contrassegnata dalle contraddizioni e piena di varie tentazioni. $\grave{E}$ il legame che collega il protagonista ai bei ricordi del passato, soprattutto a suo padre che era un seguace dello shaykh e lo accompagnava da lui

(Il vento nel vigneto di Sgorlon e Il ladro...) Dr. Fawzi Issa 
invitandolo ad ascoltare e imparare. È anche il rifugio sempre aperto e ospitale a cui il protagonista può ricorrere ogni volta che tutte le porte gli diventano chiuse davanti. Dato ciò Said Mahran appena uscito dalla prigione si dirige a questa casa per passare la notte e prendere fiato prima di cominciare la sua avventura verso la rivalsa. Anche verso la fine quando scopre che la polizia assedia la casa di Nur, egli fugge per nascondersi per l'ultima volta dallo shaykh:

«Poco prima dell'alba fa ritorno alla casa dello shaykh: questi è inginocchiato nell'angolo della preghiera. Si toglie il vestito e, nonostante la certezza di non potersi addormentare, si distende sua stuoia con volto rivolto verso il muro. Lo shaykh gli dice:

"Dormi, perché il sonno è un atto di fede, per quelli come te!" ${ }^{43}$

Alish è l'antagonista di Said Mahran, era un uomo di poco conto che si è fatto grazie a Said Mahran, diventando il suo aiutante e il suo complice, ma poi lo denuncia e lo fa arrestare, impossessandosi della refurtiva e poi anche rubandogli la moglie. È l'esempio del complice infedele e opportunista pronto a tradire il suo compagno o il suo maestro, perciò è uno dei "cani" del racconto e il primo obiettivo della vendetta del "ladro":

«Alish, non ricordi più quando ti strusciavi contro le mie gambe come un cane? Non ti ho forse insegnato io a camminare? Chi è stato a fare di un ammasso di rifiuti un uomo? Ma non solo tu hai dimenticato, se l'è scordato anche lei, quella donna perfida e indegna». ${ }^{44}$

Il padron Tarzan, invece, è l'archetipo del padrone popolano fedele all'antico amico e disposto ad aiutarlo, in ogni caso e a tutti i costi. Egli aiuta il protagonista ogni volta

(Il vento nel vigneto di Sgorlon e Il ladro...) Dr. Fawzi Issa 
che ne ha bisogno. All' inizio lo aiuta a trovare Nur e gli procura un'arma da fuoco. Spia per lui anche gli uomini di Alish e lo aiuta a tirare Baiada alla trappola. Infine, lo aiuta a scansare la polizia e gli consiglia di allontanarsi per un po' di tempo dal quartiere, visto che i poliziotti si sono diffusi nella zona.

\section{IL PERSONAGGIO FEMMINILE NELLE DUE OPERE}

Le figure femminili nelle due opere svolgono diversi ruoli pro e contro il protagonista. Nel romanzo italiano i personaggi femminili più importanti per la trama sono Iolanda, Rita e Giovanna. Quest'ultima viene presentata dall'autore come la popolana tradizionale che, malgrado era molto attaccata a Eliseo durante il processo, con il passar degli anni lo dimentica, e si perde nella quotidianità e nei problemi della vita. Appena uscito dalla prigione Eliseo era molto interessato a conoscere le sue notizie: ne chiede prima alla Sorella Iolanda e poi al cognato di Rita. Quando conosce che suo marito è morto ed è oramai vedeva decide di farle una visita, ma una volta incontrata scopre che non è Giovanna che conosceva o che pensava di conoscere: all' inizio non lo riconosce perché pensava che fosse ancora in carcere, poi gli racconta i problemi delle figlie sposate e delle sue galline malate. Non gli ha chiesto niente di lui. Così Eliseo decide di non vederla più:

«Eliseo pensò che Giovanna non gli aveva chiesto niente di lui, eppure era la stessa donna che aveva tanto pianto, alla Corte d'Assise di Udine, e nel fisico non era neanche molto cambiata... Cercò di scusarla, dentro di sé, perché sapeva oramai bene che gli uomini vivono

(Il vento nel vigneto di Sgorlon e Il ladro...) Dr. Fawzi Issa 
tutti quanti agganciati al presente e ai loro interessi immediati, e non sapevano staccarsene, come lui, ma tuttavia si sentiva molto triste. Scosse la testa, e a Giovanna cercò di non pensarci più». ${ }^{45}$

La sorella di Eliseo, Iolanda, è una delle figure più importanti del romanzo. Viene tratta fisicamente dall'autore come una donna grassa e robusta. Come carattere, invece viene presentata al lettore come l'archetipo della donna guerriera, dalla personalità forte. La sua forza è stata dimostrata durante la crisi del fratello, poiché ha dovuto lei seguire il processo e vendere la terra ed i cavalli e ipotecare la casa per le spese dell'avvocato e mantenere la famiglia: $i$ genitori anziani e l'altro fratello palizzato. Quando sono morti i genitori lei continuò a prendersi cura del fratello handicappato, persino l'ha portato con lei quando si è sposata. Ella nutriva qualche sentimento di rancore nei confronti di Eliseo che considerava la causa della rovina di tutta la famiglia.

Il primo incontro tra loro dopo ventisette anni di prigione è stato molto freddo e assai triste ed è durato molto poco. Il secondo poi incontro era più triste perché lei insisteva a fargli vedere le carte de e lei dice esplicitamente al fratello che ha dimenticato quello che era successo per colpa sua:

«Iolanda parlava solo di cifre, di vendite e ipoteche, di spese, ma sia lei che Eliseo sentivano che tra quelle carte si muovevano ancora i fantasmi del passato, la roba perduta, la casa venduta, i vecchi morti di crepacuore. Ella avvertiva con forza, come il primo giorno, che la colpa della rovina della sua famiglia era lui, lui soltanto. In ventisette anni quel sentimento forse si era sbiadito, 
ma rivedendosi il fratello davanti, rivoltando quei vecchi documenti, esso aveva riacquistato la vivacità di quando le cose erano successe». ${ }^{46}$

Un cambiamento positivo nella relazione tra Iolanda e Eliseo avviene quando quest'ultimo riesce a trovare una sistemazione e un lavoro. Quando Egli va a trovarla e lei vede che il fratello si veste bene gli fa un'accoglienza più calorosa poiché non si sente più la vergogna che nutriva prima:

«... Iolanda gli venne incontro e vedendolo vestito bene ne fu soddisfatta per qualche segreto orgoglio di famiglia. In fondo Eliseo era sempre suo fratello.

"Sono contenta di vederti" gli disse. "Come mai da queste parti?".

"Sono venuto a trovarti".

[...]

"Che lavoro hai trovato?".

Eliseo disse che girava per comprare mobili e oggetti antichi, che poi rivendeva agli antiquati di Udine, ma non nominò stracci e ferrivecchi. La parola antiquari fece un certo effetto su Iolanda, la quale pensò che il fratello non doveva guadagnare male, a giudicare da come era vestito». ${ }^{47}$

Rita è l'archetipo della donna quasi perfetta: è razionale e coraggiosa che non dà retta alle parole della gente del paese perciò dà in affitto la camera vuota a Eliseo malgrado che lui sia estraneo e ex carcerato. $\mathrm{E}$ anche l'esempio della madre disposta a far sacrifici per il proprio figlio, perciò non si lascia andare dietro al suo sentimento verso l'uomo amato. Si tratta insomma di una figura molto tenera e amabile, ma nello stesso tempo forte e intelligente che sa mantenere l' equilibrio tra il cuore e la ragione, perciò

(Il vento nel vigneto di Sgorlon e Il ladro...) Dr. Fawzi Issa 
alla fine riesce, in un certo qual modo, a rappresentare il focolare domestico mancato anche per Eliseo, malgrado abbia rifiutato la sua proposta di matrimonio per non danneggiare il futuro del figlio Riccardo:

"Sentite" gli disse "io e voi dobbiamo fare un patto".

"Sentiamo".

"Voi non avete una donna e io... io sono vedova. È un vantaggio per tutti e due se cerchiamo di autarci l'uno con l'altro. Voi fate i lavori da uomo per me, e dovete dunque lasciarmi fare quelli da donna per voi'.

"Però quello che faccio io per voi me l'avete pagato".

"D'ora in poi lo farete gratis. Allora, siamo d'accordo?"

"D'accordo" disse Eliseo». 48

I personaggi femminili nel romanzo di Nagib Mahfuz sono due, la moglie Nabawiya e la prostituta Nur, che sono gli archetipi opposti: la prima rappresenta la moglie perfida, infedele e opportunista perché quando Said Mahran è stato catturato e prigionato, lei ha chiesto il divorzio e poi si è sposata con Alish:

«Mi ha lasciato per uno dei miei aiutanti, un apprendista che si comportava con me come il cane con il padrone. Ha chiesto il divorzio con il pretesto della mia carcerazione, poi l'ha sposato."

[...]

"Non sono stato arrestato grazie all'iniziativa della polizia, nient'affatto! Ero certissimo di cavarmela, come sempre, ma quel cane mi aveva denunciato: mi aveva accusato d'accordo con lei. Poi sono iniziati i guai e, in conclusione, ecco mia figlia che mi rinnega." 49

Nur invece, malgrado sia prostituta, è innamorata di Said ed è sempre disponibile a fare sacrifici per lui. Quando è uscito dal carcere gli dà l'amore e la passione, ma anche lo

(Il vento nel vigneto di Sgorlon e Il ladro...) Dr. Fawzi Issa 
aiuta a scappare dalla polizia. Lo vuole salvare a tutti i costi ed è disposta a lasciare tutto il mondo e stare solo con lui. Insomma, rappresentava una via di salvezza per il protagonista, come lo è lo shaykh Gunaydi che lo invita a seguire la retta via, ma lui non ha voluto scegliere né l'una né l'altra, come se l'autore volesse dire che, fin dall'inizio, il suo destino era segnato.

\section{CONCLUSIONE}

In conclusione, possiamo dire che le due opere prese in esame in questo studio appaiono, apparentemente, semplici o tradizionali per la loro tematica molto attuale, ma in realtà la scorrevolezza della loro lettura è quasi un mantello che cela altre dimensioni filosofiche più profonde. E malgrado siano accumunate da una somiglianza tematica, rispecchiano due mondi assai diversi. Ognuno dei due scrittori è riuscito perfettamente a trasmettere al lettore la propria concezione filosofica della vita e presentargli la propria realtà geografica e culturale. Tale particolarità e provincialità ha procurato allo scrittore egiziano l'internazionalità incoronata poi con il premio Nobel, mentre lo scrittore italiano non ha ancora ottenuto quanto merita di stima sul livello mondiale.

Alla vicenda dell'opera di Carlo Sgorlon fa da sfondo una civiltà ormai in via di sparizione con le sue caratteristiche ed i suoi valori che l'autore mette in risalto attraverso le riflessioni e le contemplazioni del protagonista. A quella, invece, dell'opera di Nagib Mahfuz si intreccia un delicato contrappunto di riflessioni politiche, etiche, religiose affidato a volte alle parole dello Shayek, a volte alle amare

(Il vento nel vigneto di Sgorlon e Il ladro...) Dr. Fawzi Issa 
riflessioni della prostituta Nur, a volte ai pensieri del protagonista.

Gli ampi squarci metafisici delle due opere convergono, anche se in modo diverso, nel sottolineare l'angosciata solitudine dell'individuo nel mondo, la sua impossibilità di capire e controllare il senso del proprio "esserci", l'idea che la società e la Storia siano macchine sostanzialmente autosufficienti, del tutto indifferenti alla sorte dei singoli uomini che coinvolgono: una condizione priva di luce, che prende forma nei rintocchi di una scrittura tanto più tragica, quanto più asciutta e trasparente.

Infine, vorremmo sottolineare che un esame contrastivo più dettagliato e più profondo di queste due opere necessita di uno spazio più ampio di quello disponibile nel nostro studio attuale, soprattutto per quanto riguarda l'analisi dei personaggi e la dimensione metafisica e spirituale. 
${ }^{1}$ R. Damiani, Carlo Sgorlon narratore, Gremese, Roma 1978, p.14.

${ }^{2}$ C. Sgorlon, Il vento nel vigneto, Gremese Editore, Roma, 1982, p. 146.

${ }^{3}$ Ivi, p. 87.

${ }^{4}$ Ivi, p. 147.

${ }^{5}$ Bruno Maier, Carlo Sgorlon, Il Castoro n.201, Tipografia ITER, Roma, 1985 p. 12.

${ }^{6}$ Sgorlon, Il vento nel vigneto, op.cit., p. 112.

${ }^{7}$ Anonimo in C. Sgorlon, I sette veli, Arnoldo MondadoriEditore, Milano 1986, p.5.

${ }^{8}$ C. Sgorlon, Riconsacrare il mondo, testimonianza in occasione del Vesperale dell'11 marzo 1984 p:16.

${ }^{9}$ Nagib Mahfuz, Il ladro e i cani, traduzione di Valentina Colombo, Feltrinelli editore, Milano, 1990. p. 8.

${ }_{11}^{10} \mathrm{http}: / /$ www.ilgrinzone.it/il-ladro-e-i-cani.html

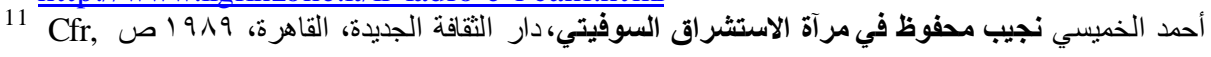
r

${ }^{12}$ C. Sgorlon, op. cit., p. 25.

${ }^{13}$ Ivi, p. 14.

${ }^{14}$ Ivi, p.99.

${ }^{15}$ Ivi, p. 101.

${ }^{16}$ Ivi, p. 142.

17 Nagib Mahfuz, op.cit., p. 19.

${ }^{18}$ Ivi, p. 11.

${ }^{19}$ C. Sgorlon, op. cit., pp. 110-111.

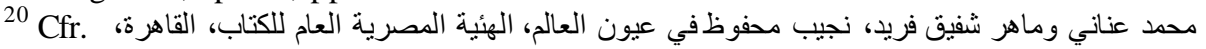
qr.

21 Nagib Mahfuz, op.cit. p. 40.

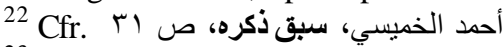

${ }^{23}$ C. Sgorlon, op. cit., p. 5.

${ }^{24}$ N. Mahfuz, op.cit., p. 7.

${ }^{25}$ C. Sgorlon, op. cit., p.8.

${ }^{26}$ Ivi, p. 38

${ }^{27}$ N. Mahfuz, op. cit., p. 9 T

${ }^{28}$ Ivi, pp. 35- 36.

${ }^{29}$ C. Sgorlon, op. cit., p.89.

30 Ivi, p. 94.

31 Ivi, p. 128.

${ }^{32}$ Ivi, p. 12.

${ }^{33}$ Ivi, p. 130.

${ }^{34}$ Ivi, p. 50.

${ }^{35}$ Mahfuz, op. cit., p.95.

36 Ivi, p. 103.

${ }^{37}$ Ivi, pp. 137-138.

${ }^{38}$ C. Sgorlon, op. cit. p. 64.

${ }^{39}$ Ivi, pp. 160- 161.

${ }^{40}$ Ivi, p. 68.

${ }^{41}$ Ivi, p. 92.

إير اهيم فتحي، العالم الروائي عند نجيب محفوظ، الهئية المصرية العام للكتاب، القاهرة، ج ا ـ r، ص Cfr, AV

(Il vento nel vigneto di Sgorlon e Il ladro...) Dr. Fawzi Issa 
${ }^{43}$ N. Mahfuz, po.cit p. 134.

${ }^{44}$ Ivi, p. 8.

${ }^{45}$ Sgorlon, op. cit., p. 109.

${ }^{46}$ Ivi, p.54.

${ }^{47}$ Ivi, p. 156.

48 Ivi, p. 114.

${ }^{49}$ N. Mahfuz, op.cit.,p. 25. 


\section{Bibliografia}

- Alberti, Carmelo, L'opera narrativa di Carlo Sgorlon, Bastogi editore, Foggia, 2003.

- Chines, Loredana e Varotti, Carlo, Che cos'è un testo letterario, Carocci editore, Roma 2015.

- Damiani, Roberto, Carlo Sgorlon narratore, Gremese Editore, Roma, 1978.

- Di Monaco, Bartolomeo, Omaggio a Carlo Sgorlon, Marcovalerio editore, 2015.

- Ghidina, Jean Igor, Mito, società e scrittura nell'universo romanzesco di Carlo Sgorlon, La nuova base editrice, 2006.

- Mahfuz, Nagib, Il ladro e i cani, traduzione di Valentina Colombo, Feltrinelli editore, Milano, 1990.

- Maier, Bruno, Carlo Sgorlon, Il Castoro n.201, Tipografia ITER, Roma, 1985.

- Nissim, Liana, Sgorlon teste insolente, Edizioni del Camajun, Como, 1985.

- Sgorlon, Carlo, Il vento nel vigneto, Gremese Editore, Roma 1982.

- Sgorlon, Carlo, RICONSACRARE IL MONDO, in occasione del Vesperale dell' 11 marzo 1984, Edizione fuori commercio, esemplari da 1 a 500, esemplare n. 154 .

- Sgorlon, Carlo, I stte veli, Arnoldo Mondadori Editore, Milano 1986.

- Toscani, Claudio, Invito alla lettura di Carlo Sgolon, Mursia, Milano, 1987.

\section{PERIODICI:}

- Ferdinando Castelli, L'odissea drammatica dei cosacchi in un romanzo di C. Sgorlon, S. I. Civilta' Cattolica, 20-7-85, p.132.

- Gino, A., quel capolinea c'è un'illusione, Gazzetta del popolo, 11-51979. 


\section{المصادر العربية}

إبر اهيم فتحي، العالم الروائي عند نجيب محفوظ، الهئية المصرية العام للكتاب،

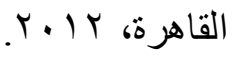

أحمد الخميسي، نجيب محفوظ في مرآة الاستشراق السوفيتي، دار التقافة الجديدة،

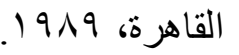

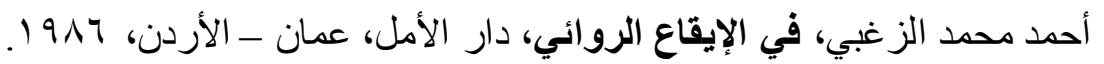

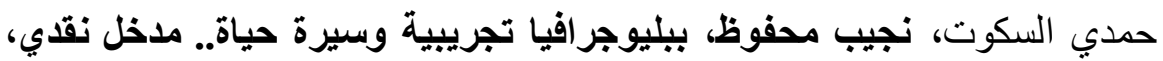

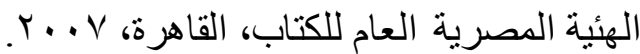
سامي سليمان أحمد، مدخل إلى دراسة النص العربي المعاصر.. قراعات نقدية

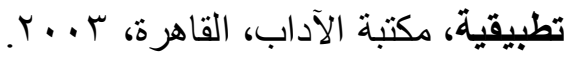
عبد الصمد زايد، مفهوم الزمن ودلالته في الرواية العربية المعاصرة، الدار

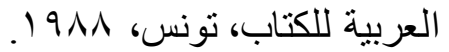
عبد الغفار رشدي، بين الأدب والاراما، مركز الأهرام للنشر والترجمة والتوزيع، تونه

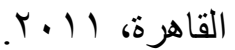
غالي شكري، المنتمي.. دراسة في أدب نجيب محفوظ، دار أخبار اليوم، القاهرة .1911 فاطمة موسى، بين أدبين، مكتبة الأنجلو المصرية، القاهرة 970 أمهرة لطيفة الزيات، نجيب محفوظ الصورة والمثال، الهئية المصرية العام للكتاب،

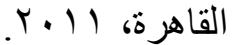
نبيل راغب، قضية الثكل الفني عند نجيب محفوظ، دار الكتاب العربي، القاهرة، $.197 \mathrm{~V}$ نجيب محفوظ، اللص والكلاب، مكتبة مصر، القاهرة الو 197 . محمد حسن عبد الله، الإسلامية والروحية في أدب نجيب محفوظ، مكتبة الأمل،

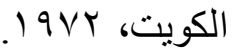
محمد عناني وماهر شفيق فريد، نجيب محفوظ في عيون العالم، الهئية المصرية

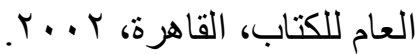
محمود الربيعي، قراءة الرواية.. نماذج من نجيب محفوظ، دار المعارف، القاهرة، 


\section{Sitografia}

https://cbmaarifa.blogspot.com/2016/07/blog-post_43.html

https://www.bartolomeodimonaco.it/online/letteratura-carlo-sgorlon-

$\% \mathrm{E} 2 \% 80 \% 9$ Cil-vento-nel-vigneto\%E2\% 80\%9D-1960/

http:/www.lafrusta.net/rec_sgorlon.html

https://ilbuonlettore.wordpress.com/2015/07/24/il-vento-nel-vigneto-di-carlo-

sgorlon-recensione/

https://www.italialibri.net/dossier/sgor lon/attivita.html

http//www.italialibri.net/dossier/sgorlon/attivita.html

https://it.wikipedia.org/wiki/Carlo_Sgorlon

http:/www.sgorlon.it/

http:/www.repubblica.it/spettacoli-e-

cultura/2009/12/26/news/addio_a_carlo_sgorlon_narratore_della_terra_friulan a-1822588/

https://it.wikiquote.org/wiki/Carlo_Sgorlon

(Il vento nel vigneto di Sgorlon e Il ladro...) Dr. Fawzi Issa 
رواية الرياح في بستان العنب لـ سجورلون ورواية اللص والكلاب لمحفوظ: مقارنة

\section{بين عالمين \\ ملخص}

تهدف هذه الدراسة إلى المقارنة بين عالم الأديب الإيطالي كارلو سجورلون

في رواية "الرياح في مزرعة العنب" وعالم الأديب المصري نجيب محفوظ في روايته "اللص والكلاب"، وهما عالمان مختلفان تماما إلى حد النتاقض. والروايتان اللتان كُتبتا تقريبا في نفس التوقيت تتتاولان تقريبا الموضوع ذاته، وهو تجربة شخص خرج لتوه من السجن ومحاولته العودة إلى حياته السابقة وطريقته في مواجهة المجتمع أو الانخراط فيه. ومع ذلك فإن كل واحدة من الروايتين تعكس عالم وفكر وفن مؤلفها. فرواية سجورلون التي كتبها عام • ب91، وإن لم يتم نشرها إلا في عام بلو 1، تروي قصة إليزيو الذي يعود، بعد أن خرج من السجن، إلى الأماكن التي ولد وعاش فيها من قبل، ويحاول بإصرار الانخراط في المجتمع بالرغم من الصعوبات ورفض المجتمع في البداية. وبفضل نضجه داخل السجن وتطور شخصيته واكتسابه للحكمة، ينجح البطل في النهاية في تحقيق ذاته والتصالح مع المجتمع ومع نفسه. وعلى عكس بطل الرواية الإيطالية، فإن سعيد مهران بطل "اللص والكلاب" يخرج من السجن وليس في ذهنه سوى هدف واحد وهو الانتقام. وبقدر ما نجح إليزيو في تحقيق هدفه، بقدر ما فنل سعيد مهران في كل شيء، فقد فنل في الانخراط في المجتمع وفثلت كل محاولاته للانتقام من خصومه، فهو مثال للبطل السلبي، ولم ينجح سوى في رسم رؤية كاتبه في كثف النفاق والرياء والزيف الذي تتصف به شريحة واسعة من المجتمع. إنهما شخصيتان منتاقضتان تعكسان عالمين منضادين، ولكنهما تعبران بدقة عن فكر

مؤلفيهما. كلمات مفتاحية: سجورلون - محفوظ - الرياح - بستان العنب - اللص والكلاب (Il vento nel vigneto di Sgorlon e Il ladro...) Dr. Fawzi Issa 